and a review of systems in only seven. A clear statement of the indications for treatment with lithium was found in 18 of the notes, while an explicit treatment plan had been preserved in only five.

In none of the case notes was there a record suggesting that the patient had been informed of the possible side effects or toxicity of lithium, nor of the need to have regular blood tests and to maintain adequate fluid intake. Lithium is known to be a toxic drug and patients are undoubtedly given information about its use, but this should be recorded, particularly in view of recent medicolegal interest in the use of lithium.

Kehoe and Mander comment that certain aspects of lithium prescribing and monitoring habits give cause for concern; our audit suggests that this concern should also be extended to the period when lithium is first prescribed and to the recording of treatment and advice given.

J $S$ E HELLEWELL ELIZABETH WYN PUGH

Department of Psychiatry, North Manchester Health Authority,

Manchester M86RB

1 Kehoe RF, Mander AJ. Lithium treatment: prescribing and monitoring habits in hospital and general practice. $B M \mathcal{J}$ 1992;304:552-4. (29 February.)

\section{Is Bordetella pertussis clonal?}

SIR, - M N Khattak and colleagues were right to finish their title with a question mark. ${ }^{\prime}$ Although their elegant study shows the discriminatory power of genotype analysis by restriction enzyme digestion and pulsed field gel electrophoresis and its suitability for typing and epidemiological tracing, we do not agree with the conclusion that their results fail to support the hypothesis that this organism is essentially clonal.

The operational definition of clones provided by Ørskov and Ørskov describes them as "bacterial cultures isolated independently from different sources, in different locations and perhaps at different times, but showing so many identical phenotypic and genetic traits that the most likely explanation for this identity is a common origin." Implicit in this concept is the requirement that the specific combinations of chromosomal genes coding for characters identifying clones are not rapidly broken down by recombination and mutation. ${ }^{3}$ The authors' demonstration that six of the seven DNA types found in German strainsaccounting for 22 of the 23 strains studied-were also found in the United Kingdom indicates to us that for these markers the rates of change caused by recombination and mutation are slower than the rates of spread of these organisms through western Europe, thus leading to the occurrence of statistically overrepresented, widespread, identical genotypes, a coincidence of features which is a strong criterion of clonality. ${ }^{+}$Our view is that rather than refuting the clonal concept for Bordetella pertussis the pulsed field gel electrophoresis data provide quite good evidence favouring the hypothesis that in genetic terms this organism has a relatively stable, and clonal, population structure.

The inability of simple electrophoresis of restriction fragments of whole DNA produced by Eco RI, $S m a \mathrm{I}, N c i \mathrm{I}, B a m \mathrm{HI}, A v a \mathrm{I}$ and $B g l \mathrm{II}$ to discriminate between strains suggests that they are very closely related. Whether these European strains are members of a single clone with pulsed field gel electropherotypes defining subclones, or whether these define clones in themselves, cannot be answered from the data to hand; information on the extent of linkage disequilibrium and further studies using other markers of genotype and phenotype would clarify this situation. It seems to us that the authors have answered the question posed by the title of their paper in the affirmative; the really important question that remains - albeit one that they have begun to address - is whether whooping cough in Britain and Germany is caused by one, or several, clones of $B$ pertussis.

T H PENNINGTON

K J FORBES

Department of Medical Microbiology,

University of Aberdeen,

Aberdeen AB9 2ZD

1 Khattak MN, Matthews RC, Burnie JP. Is Bordetella pertussis clonal? BMF 1992;304:813-5.

2 Ørskov F, Ørskov I. Summary of a workshop on the clone concept in the epidemiology, taxonomy and evolution of the Enterobacteriaceae and other bacteria. F Infect Dis 1983;148: Enterob.

3 Milkman R, Bridges MM. Molecular evolution of the $E$ coli chromosome. III. Clonal frames. Genetics 1990;126:505-17.

4 Tibayrenc M, Kjellberg F, Arnaud J, Oury B, Breniere SF, Darde $M-L$, et al. Are eukaryotic microorganisms clonal or sexual? A population genetics vantage. Proc Natl Acad Sc USA 1991;88:5129-33.

AUTHORS' REPLY,-T H Pennington and K J Forbes suggest that the 17 distinct DNA types generated by pulsed field gel electrophoresis identify "subclones" and that Bordetella pertussis is essentially stable and clonal. It is unclear as to how they make the distinction between clones and subclones. Phenotypically $B$ pertussis is not particularly stable. It shows striking colonial variation on repeated subculture ${ }^{1}$ changes in agglutinogen type occur both during infection and in response to vaccination $;^{2}$ and phase variation (loss of virulence factors) occurs at a frequency of one per $10^{3}$ or $10^{6}$ organisms. ${ }^{13}$

The types isolated by pulsed field gel electrophoresis that we describe were stable on repeated subculture and persisted after cross infection between siblings, yet they were clearly distinguishable in the four different pairs of siblings. In the light of these distinct, stable genetic differences it seems difficult to argue the case for clonality. Pennington and Forbes cite "the occurrence of statistically overrepresented, widespread, identical genotypes" as a strong criterion of clonality, yet we showed that the predominant genotype in the United Kingdom was not found among the 23 isolates examined from Germany.

R C MATTHEWS

J P BURNIE

Department of Medical Microbiology,

University of Manchester Medical School,

Manchester M13 9PT

1 Peppler MS. Isolation and characterization of domed hemolytic and flat non-hemolytic colony types of Bordetella pertussis. Infect Immun 1982;35:840-51.

2 Preston NW. Pertussis today. In: Wardlaw AC, Parton R, eds. Pathogenesis and immunity in pertussis. Chichester: Wiley, 1988:1-18.

3 Weiss AA, Falkow S. Genetic analysis of phase changes in Bordetella pertussis. Infect Immun 1984;43:263-9.

\section{Physiotherapy intervention late after stroke}

SIR, - In their paper on the effectiveness of physiotherapy on mobility late after stroke, Derick T Wade and colleagues went to great pains to develop a cross over study design which introduced blind assessment and controls. ${ }^{1}$ This attention to design is not typical of research in stroke rehabilitation and the authors are to be congratulated. However, the results are ambiguous.

The study showed that mobility (as measured by time to walk 10 metres) decreased by about four seconds during the treatment phase but increased after treatment. This would be expected if the physiotherapy intervention was very active and required regular and specialised exercises. However, table II shows that much of the intervention was of an advisory and educational nature, including the provision of more appropriate aids. It is therefore worrying to note a decline in mobility in the period immediately after treatment in both groups $(6.5$ seconds in the early treatment group and 2.6 seconds in the late treatment group). The fact that these increases are not statistically significant is due to the acknowledged low power of the study.

These results strongly suggest that the improvement during treatment might be a placebo effect due purely to the attention given by the physiotherapist rather than the direct result of the interventions mentioned. The fact that the possible placebo effect was confined to changes in gait speed is probably a reflection of the lack of sensitivity to change of the other outcome measures, as is acknowledged by the authors.

A cross over design in drug treatment trials is invariably associated with the use of either a placebo or an alternative treatment. In such trials the differences in outcome between the treatment period and non-treatment period can be more confidently attributed to the treatment. In this study, because patients received no treatment or placebo in the non-treatment period it is impossible to disentangle the treatment effects of physiotherapy from any placebo effects.

Physiotherapy consists of more than direct physical treatment, also having a significant psychological component. Therefore the authors must be careful when they attribute the positive outcome to the more physical aspects of the intervention, for it is possible that a similar outcome could have been achieved by using other personnel such as well motivated and supervised volunteers, as has been shown in speech therapy after stroke. ${ }^{2}$

Although we recognise that finding placebos for physical and counselling interventions is much more difficult than in pharmacological areas, it is important that attempts are made to develop ways of controlling for placebo effects in an area where attention and care are likely to have significant beneficial effects on patients' performance.

TREVOR A SHELDON NICK D FREEMANTLE COLIN T POLLOCK

School of Public Health

University of Leeds,

Leeds LS2 9LN

1 Wade DT, Cullen FM, Robb GF, Warlow CP. Physiotherap intervention late after stroke and mobility. BMF 1992;304: 609-13. (7 March.)

2 Freemantle N, Pollock C, Sheldon TA. Formal rehabilitation after stroke-a review of effectiveness. Quality in Health Car (in press)

AUTHOR'S REPLY,-Trevor A Sheldon and his colleagues should reread the first sentence of our abstract: "To determine whether the intervention of a physiotherapist improved mobility.

Rehabilitation is a problem-solving, educationa process aiming to minimise handicap. Its reiterative components include assessment, planning (goal setting), intervention, and evaluation (reassessment) and each of these can be further subdivided. Few components of this process can be so isolated that effects can be attributed to one specific item We were very careful to state throughout that the trial investigated the effect of being seen by a physiotherapist.

Next, if attention alone had caused improvement in gait speed why was no change seen in manual dexterity or mood? Why should attention specifically and only improve gait speed?

One cannot assume that volunteers might be as effective without further trials. The volunteers in speech therapy trials were given the results of assessments and were supervised by trained therapists. The equivalent would be for the first "assessment" visits to be undertaken by a trained therapist, followed by visits by volunteers with trained therapists supervising. As the average number of visits was four, this seems uneconomic. 